\title{
Dielectric properties: a gateway to antibacterial assay-a case study of low-density polyethylene/ chitosan composite films
}

\author{
Madathil Sunilkumar ${ }^{1}$, Ambalakkandy Abdul Gafoor ${ }^{2}$, Abdulaziz Anas ${ }^{3}$, Areepuravan Parakkal Haseena ${ }^{1}$ \\ and Athiyanathil Sujith ${ }^{1}$
}

The dielectric properties of low-density polyethylene-chitosan composite films were correlated with their antibacterial properties in this work. Films were designed on the molecular level using palm oil as a plasticizer in an internal mixer. Maleic anhydride and dicumyl peroxide were used as a coupling agent and a free radical initiator, respectively. The dielectric properties of the composite films were studied as a function of chitosan loading, presence of plasticizer and variable applied frequency. The dielectric constant of the samples was positively correlated with the amount of chitosan in the polythene matrix. The antibacterial properties of the composite films were also studied. A distinct inhibitory zone against Escherichia coli and Staphylococcus aureus developed, and this zone increased in diameter with chitosan loading. The low-molecular-weight chitosan-based polyethylene films showed significant improvement in dielectric and antibacterial properties when compared with the native chitosan-based films. These studies show that the antibacterial properties of the developed films were complementary with their dielectric properties. This study suggests the possibility of using the dielectric properties of composites as a measure of their antibacterial properties.

Polymer Journal (2014) 46, 422-429; doi:10.1038/pj.2014.19; published online 23 April 2014

Keywords: antimicrobial activity; chitosan; dielectric properties; FESEM

\section{INTRODUCTION}

Antimicrobial food packaging has been an area of interest for many years because it can enhance the safety and quality of food. ${ }^{1-4}$ It is a promising method to prevent both the contamination from pathogens and growth of microorganisms that cause spoilage on the surface of food products. ${ }^{5,6}$ A simple and low cost method of producing both eco-friendly and antimicrobial film is to incorporate an antimicrobial component into a suitable matrix. Cellulose and chitosan are commonly used for the synthesis of biopolymer composites, which are effective carriers of antimicrobial agents. ${ }^{7,8}$ Among these biopolymers, chitosan exhibits inherent antimicrobial properties. ${ }^{9,10}$ Chitosan does not require a carrier, and it can also be used as a coating or can be cast into polymer films with good strength, barrier properties and biocompatibility.

The polycationic nature of chitosan interferes with the negatively charged cell membrane of pathogens and causes membrane leakage, which can prevent microbial growth by prohibiting their DNA transformation and metabolic activity. ${ }^{11}$ A systematic investigation of the dielectric properties of the polymeric composite films can reveal the extent of antimicrobial activity of such films. The dielectric properties of various polymeric systems have been investigated by many researchers. ${ }^{12-14}$ It has been reported that the dielectric properties of polymeric materials depend on the chemical structure, crystallinity, morphological features and presence of fillers or additives. The dielectric constant of a material increases with the effective dipole moment. ${ }^{14}$ It has also been reported that the introduction of polar components into the polyethylene matrix could increase its dielectric constant and dielectric loss. ${ }^{15}$ In heterogeneous polymer blends, the dielectric constant of the polymers is influenced by interfacial effects from the polarization arising from the difference in conductivities of the two phases. ${ }^{15}$ The volume resistivity of various polymeric systems upon the incorporation of fillers has also been studied by researchers. ${ }^{16,17}$ Chitosan can be made into biocomposite films for food packaging because of its good mechanical properties, its ability to act as an oxygen barrier and its antimicrobial activities. The antibacterial and antifungal properties of chitosan, arising from its polycationic nature, are well known against a variety of bacteria and fungi. ${ }^{18,19}$

Low-density polyethylene (LDPE) is a low cost material that is highly processable and has excellent electrical insulation properties,

${ }^{1}$ Materials Research Laboratory, Department of Chemistry, National Institute of Technology Calicut, Calicut, India; ${ }^{2}$ Department of Physics, Government Arts and Science College Kozhikode, Calicut, India and ${ }^{3}$ Council of Scientific and Industrial Research-National Institute of Oceanography, Regional Centre Cochin, Cochin, India

Correspondence: Dr A Sujith, Materials Research Laboratory, Department of Chemistry, National Institute of Technology Calicut, Chathamangalam, NITC, Calicut, Kerala 673601, India.

E-mail: athiyanathil.sujith@gmail.com

Received 5 November 2013; revised 20 February 2014; accepted 21 February 2014; published online 23 April 2014 
good chemical resistance, high flexibility and low water vapor permeability. ${ }^{20,21}$ LDPE-chitosan composite films were developed in this work using palm oil as a plasticizer. This plasticizer is a renewable resource that is a biodegradable, non-toxic and effective coupling agent. The morphological, structural, mechanical and thermal properties of the developed films were previously investigated and reported. ${ }^{22}$ The mechanical, thermal and biodegradation properties of plasticized films improved compared with the unplasticized composites. The optimum value of tensile strength and elastic modulus of the samples were found to be 11.77 and $86.36 \mathrm{MPa}$, respectively, at a $10 \mathrm{wt} \%$ of chitosan loading in the LDPE matrix. ${ }^{22}$

The current work is the first attempt to correlate the dielectric properties and antibacterial properties of these biocomposite films. The observations will be helpful for food packaging applications by assessing the extent of antibacterial activity through measuring the dielectric properties in biocomposite films.

\section{EXPERIMENTAL PROCEDURE}

\section{Materials}

The film-grade LDPE (with a density of $0.923 \mathrm{~g} \mathrm{~cm}^{-3}$, a melt index of $6.0 \mathrm{~g}$ per $10 \mathrm{~min}$ and a softening point of $87.22^{\circ} \mathrm{C}$ ) was supplied by Reliance Industries Limited, Mumbai, India. Powdered chitosan was obtained from India Sea Foods, Kochi, India, with a degree of de-acetylation of $80.2 \%$ and a molecular weight of $3.0200 \times 10^{5} \mathrm{~g} \mathrm{~mol}^{-1}$. The chitosan was dried at $100^{\circ} \mathrm{C}$ for $5 \mathrm{~h}$ before mixing. Refined grade palm oil (with a density of $0.924 \mathrm{~g} \mathrm{~cm}^{-3}$ ) was provided by Parisons Pvt Ltd KINFRA Park, Malappuram, India. All other reagents, such as dicumyl peroxide and maleic anhydride, were analytical grade and obtained from Sigma-Aldrich, Mumbai, India.

\section{Sample preparation}

Preparation of de-acetylated low-molecular-weight chitosan (LWCS). Dried prawn chitosan flakes were added to a $1 \%(\mathrm{~W} / \mathrm{V})$ acetic acid solution in a reaction vessel and allowed to dissolve while being stirred for $30 \mathrm{~min}$. The reaction mixture was precipitated with $50 \%(\mathrm{~W} / \mathrm{V}) \mathrm{NaOH}$. The resulting suspension was then stirred at 3600 r.p.m. at $160^{\circ} \mathrm{C}$ for 5 h. The mixture was then diluted with distilled water followed by centrifugation. Next, the suspension was transferred to a dialysis bag and dialyzed in running water for $2 \mathrm{~h}$. The suspension was then dialyzed overnight in distilled water until a $\mathrm{pH}$ of 7 was reached. The chitosan precipitate was rinsed with distilled water, freeze dried and analyzed. The degree of de-acetylation of the resulting chitosan powder was measured to be $94.4 \%$ using UV-Vis spectrophotometry, Systronics, Ahmadabad, India, Model No. 2202.

The de-acetylated chitosan was mixed in $0.1 \mathrm{M} \mathrm{HCl}$ in a reaction vessel and stirred for $30 \mathrm{~min}$. Then, $15 \% \mathrm{H}_{2} \mathrm{O}_{2}$ was poured in to the reaction mixture. The solution was heated at $65^{\circ} \mathrm{C}$, stirred at 3600 r.p.m. for $2 \mathrm{~h}$ and then filtered. Two molar $\mathrm{NaOH}$ solution was added to the filtrate to achieve a $\mathrm{pH}$ of 7 , and excess ethanol was added to precipitate the product. The chitosan precipitate was dialyzed in running water for $2 \mathrm{~h}$, then it was dialyzed overnight in distilled water. Finally, the dialyzed chitosan precipitate was freeze dried and analyzed. The molecular weight of the chitosan was measured using the viscometry method. In this study, the molecular weight of the chitosan was reduced to $1.473 \times 10^{3} \mathrm{~g} \mathrm{~mol}^{-1}$ from the initial value of $3.0200 \times$ $10^{5} \mathrm{~g} \mathrm{~mol}^{-1}$. The microscopic images of the native chitosan and the deacetylated LWCS particles are shown in Figures 1a and b respectively. The native chitosan appeared as flake-like structures, whereas its de-polymerized sample appeared spherical with a considerable reduction in size.

Preparation of the composite films. The compounds were prepared in a Thermo HAAKE Polylab System (Karlsruhe, Germany) equipped with the roller-type rotors. Mixing was performed at a rotor speed of 60 r.p.m. and a temperature of $140^{\circ} \mathrm{C}$. The LDPE was first allowed to melt for $3 \mathrm{~min}$. The dicumyl peroxide, maleic anhydride and varying amounts of the biofiller native chitosan $(0,5,10$ and $15 \mathrm{wt} \%$, which were abbreviated as A1, A2, A3 and A4) were added into the LDPE matrix in 2-min time intervals for each sample. Mixing was then continued for another $4 \mathrm{~min}$. The composites obtained from the melt mixing were compressed using an electrically heated hydraulic press for $3 \mathrm{~min}$ at $150{ }^{\circ} \mathrm{C}$ under a pressure of $200 \mathrm{~kg} \mathrm{~cm}^{-2}$. After pressing, the samples were allowed to cool at room temperature. The mechanism and role of dicumyl peroxide and maleic anhydride in the fabrication of composite films are shown in Figure 2.

A second set of composite films (abbreviated as B1, B2, B3 and B4) containing fixed amounts palm oil, which were added before the addition of the cross-linker, were fabricated and tested. The amount of palm oil in the matrix was optimized as $2.5 \mathrm{wt} \%$. Plasticized composite films based on the LWCS were also prepared under the same formulations and conditions. The 5, 10 and $15 \mathrm{wt} \%$ LWCS composite films were abbreviated as nB2, nB3 and nB4, respectively. The formulation of the fabricated composite films is given in Table 1.

\section{Morphology}

The morphology was investigated by field emission scanning electron microscopy (FESEM) using Hitachi SU6600 (Tokyo, Japan) FESEM. The samples were sputter coated with gold to avoid subsequent charging before analysis by FESEM.

\section{Dielectric properties}

Dielectric measurements were carried out at room temperature using an impedance analyzer. The impedance was measured with HOIKY 3532-50 LCR HI-Tester interfaced to a computer by applying alternating current (AC) signals across the film with blocking silver electrode. The bridge was set to measure the impedance and phase angle from $42 \mathrm{~Hz}$ to $5 \mathrm{MHz}$. The dielectric constant and AC conductivity were calculated from the measured data using the dimensions of the film. ${ }^{23}$

\section{Antimicrobial activities}

The antimicrobial activity of the composite films was tested using the agar diffusion method according to Pranoto et al..$^{24}$ The composite films were cut

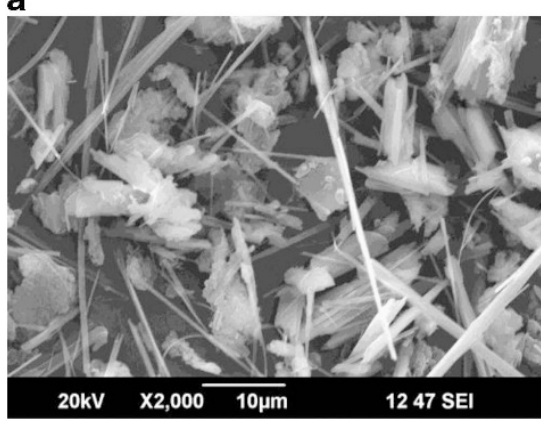

b

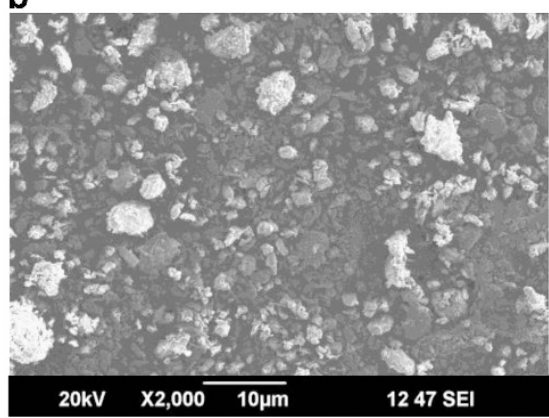

Figure 1 Scanning electron micrographs of native chitosan (a) and de-acetylated low-molecular-weight chitosan (LWCS) (b) particles. 


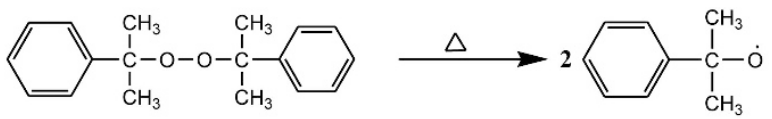

Dicumyl peroxide

Cumyl peroxide radical

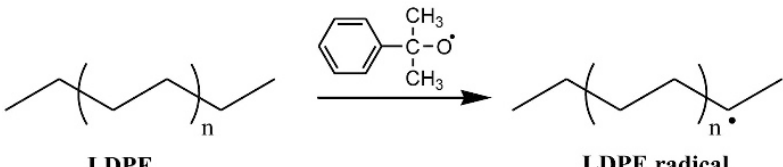

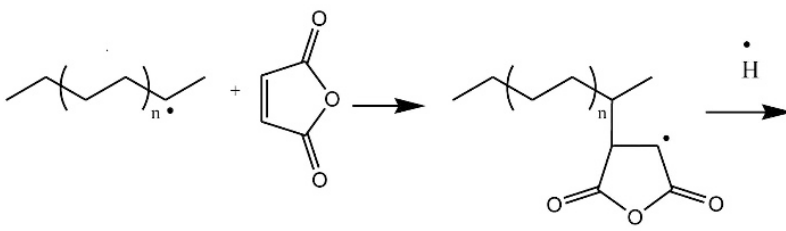

Maleic anhydride<smiles>CCCCC(C)C1CC(=O)OC1=O</smiles>

MA-g-LDPE

Figure 2 The mechanism of maleic anhydride grafting on low-density polyethylene (LDPE) using dicumyl peroxide as a free radical initiator.

Table 1 Formulation of the composite films

\begin{tabular}{lccccc}
\hline Sample & $\begin{array}{c}\text { LDPE } \\
\text { (gm) }\end{array}$ & $\begin{array}{c}\text { Chitosan } \\
\text { (Wt. \%) }\end{array}$ & $\begin{array}{c}\text { DCP } \\
\text { (Wt. \%) }\end{array}$ & $\begin{array}{c}\text { MA } \\
\text { (Wt. \%) }\end{array}$ & $\begin{array}{c}\text { Palm oil } \\
\text { (Wt. \%) }\end{array}$ \\
\hline A1 & 100 & - & 0.5 & 2 & - \\
A2 & 100 & 5 & 0.5 & 2 & - \\
A3 & 100 & 10 & 0.5 & 2 & - \\
A4 & 100 & 15 & 0.5 & 2 & - \\
\hline
\end{tabular}

Abbreviations: DCP, dicumyl peroxide; LDPE, low-density polyethylene; MA, maleic anhydride.

into $10 \mathrm{~mm}$ diameter discs and placed on Mueller Hinton agar plates (Merck, Darmstadt, Germany). These had been previously seeded with $0.1 \mathrm{ml}$ of inoculums containing tested bacteria. The plates were then incubated at $37^{\circ} \mathrm{C}$ for $24 \mathrm{~h}$. The diameter of the inhibitory zone surrounding the film discs and the contact area of the composites with the agar surfaces were noted. Experiments were performed in triplicate.

\section{RESULTS AND DISCUSSION}

\section{Dielectric properties}

Dielectric measurements were performed on the samples to study the molecular behavior of polymeric materials, which is very important in the area of effective packaging. The charge interaction between the film surface and the microbial organism determine the antimicrobial properties of the packing materials. ${ }^{25,26}$ The dielectric constant $\varepsilon_{r}$ can be determined from the capacitance using equation (1)

$$
\varepsilon_{r}=\frac{\mathrm{CL}}{\varepsilon_{0} A}
$$

where $C$ is the capacitance, $L$ the sample thickness, $\varepsilon_{0}$ the permittivity of air $\left(8.85 \times 10^{-12} \mathrm{Fm}^{-1}\right)$ and $A$ is the cross-sectional area of the samples. The dielectric constant of a composite is contributed by the interfacial, orientation, atomic and electronic polarizations of the component molecules. ${ }^{23}$ The interfacial polarization occurs in a composite due to the differences in the conductivities or the polarizations of the matrix and fillers. ${ }^{27}$ When polymers containing polar groups are placed in an electric field, polarization occurs because of the orientation of the dipoles. The orientation and

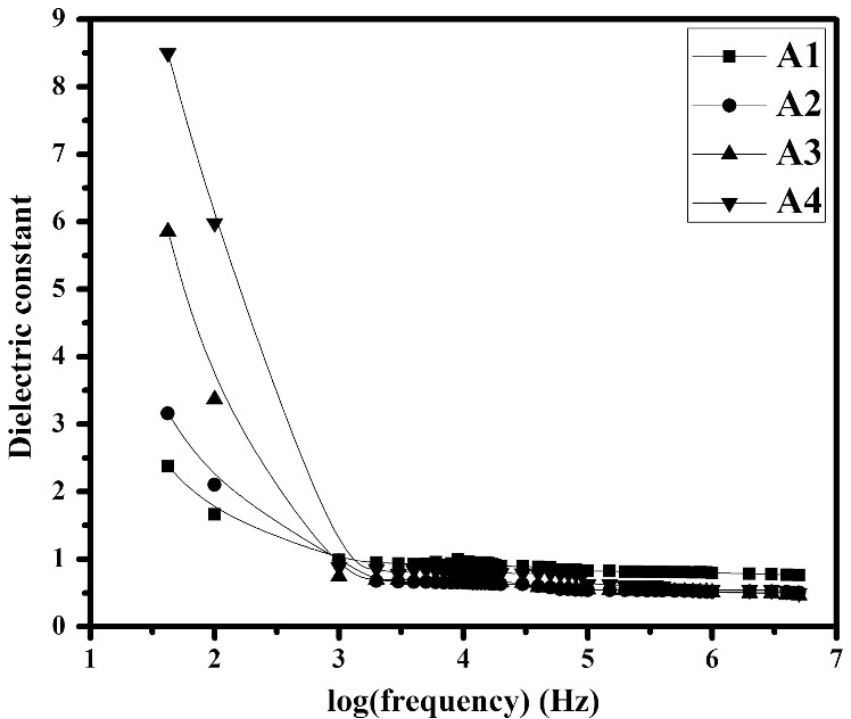

Figure 3 The effect of filler loading on the dielectric constant as a function of frequency. (A1) pure low-density polyethylene (LDPE), (A2) LDPE/5 wt\% chitosan, (A3) LDPE/10 wt\% chitosan and (A4) LDPE/15 wt\% chitosan composite films.

interfacial polarization of a composite will mainly depend upon the concentration of the fillers. ${ }^{27}$

Figure 3 shows the variation of the dielectric constant and loss with applied frequency for various unplasticized composite films. In Figure 3, A1, A2, A3 and A4 represent biocomposites with 0, 5, 10 and $15 \mathrm{wt} \%$ chitosan, respectively. It can be observed from the figure that the pure LDPE exhibited the lowest dielectric constant $\left(\varepsilon_{r}\right)$. This is due to the absence of permanent dipoles in the polythene matrix. The $\varepsilon_{r}$ values increased with chitosan loading in the composites because the dipoles increased as the weight percentage of the fillers increased in the matrix. This $\varepsilon_{r}$ increase had been attributed to the increment in orientation and interfacial polarizations resulting from the presence of polar $\mathrm{NH}_{3}{ }^{+}$ions in chitosan. It can also be observed from Figure 3 that the dielectric constant decreased with increasing 
frequency. The complete orientation of molecules required more time to reach the equilibrium static field value compared with the electronic and atomic polarizations. Therefore, the rotational motion of the polar molecules of the dielectric is not sufficiently rapid to attain equilibrium with the field as the frequency increased. ${ }^{28}$ Figure 4 shows the dielectric constant of the plasticized composite films, and it follows the same trend as the unplasticized films. An overall increase in the dielectric constant for plasticized samples was observed compared with the unplasticized samples.

Plasticizer molecules are relatively small in size compared with polymer matrix molecules. The plasticizer molecules can easily penetrate into the polymer matrices, causing an interaction between the plasticizer molecule and the polymer chain. This may reduce the cohesive forces operating between the polymer chains, resulting in an increase in the chain segmental mobility. When dipoles are directly attached to the chain, their dipole moment depends on the segmental motion of the polymeric chain. ${ }^{23}$ Hence, the incorporation of the palm oil plasticizer in LDPE-chitosan composites reduces rigidity, which increases segmental mobility and increases the dielectric

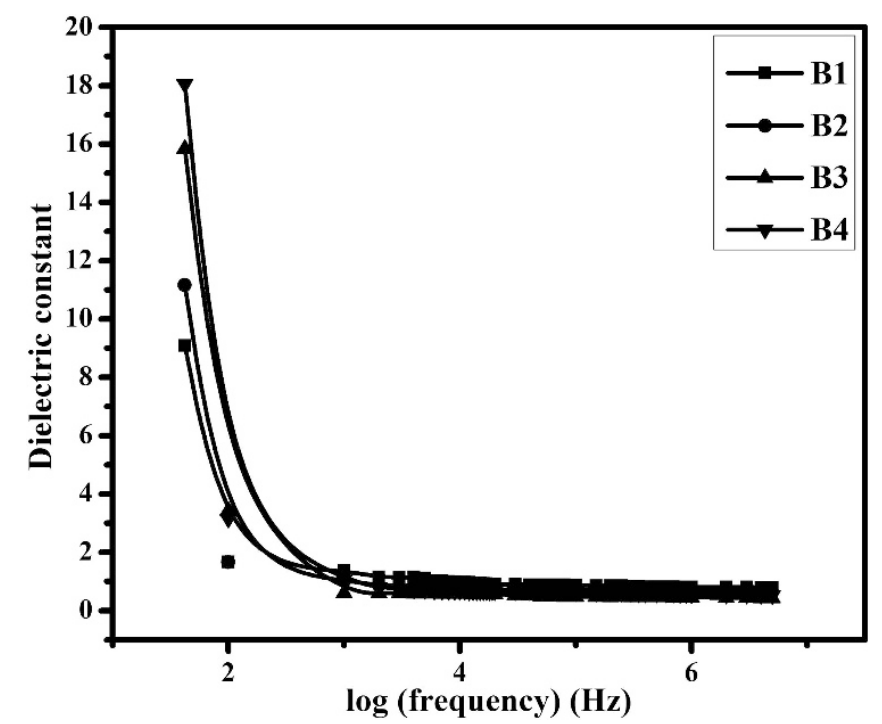

Figure 4 The effect of filler loading on the dielectric constant of the plasticized film as a function of frequency. (B1) pure low-density polyethylene (LDPE), (B2) LDPE/5 wt\% chitosan, (B3) LDPE/10 wt\% chitosan and (B4) LDPE/15 wt\% chitosan composite films. constant. The scanning electron micrographs of the unplasticized and plasticized composite films with $15 \mathrm{wt} \%$ chitosan loading are shown in Figures $5 \mathrm{a}$ and $\mathrm{b}$, respectively. The plasticized composite film (Figure 5b) shows a relatively more uniform filler dispersion compared with its unplasticized congener (Figure 5a). Owing to the presence of the palm oil plasticizer, the polythene matrix became more flexible and allowed the chitosan particles to be distributed more uniformly when compared with the rigid unplasticized matrix. Thus, the morphological study of the developed composites supported the dielectric properties.

The electrical conductivity $(\sigma)$ of composite films was determined using the following equation:

$$
\sigma=\omega \varepsilon_{0} \varepsilon_{r} \tan \delta
$$

where $\omega$ is the angular frequency that is equal to $2 \pi \gamma, \gamma$ is the applied frequency and $\tan \delta$ is the dissipation factor. Figure 6 shows the dependence of the AC electrical conductivity on chitosan loading at different frequencies. LDPE has excellent insulation with a larger band gap, a lower concentration of free charge carriers and a smaller carrier mobility compared with LDPE-chitosan films. Therefore, the effects of electrical conductivity $\sigma$ can be ignored in LDPE. Upon increasing the chitosan concentration and frequency, the AC conductivity also

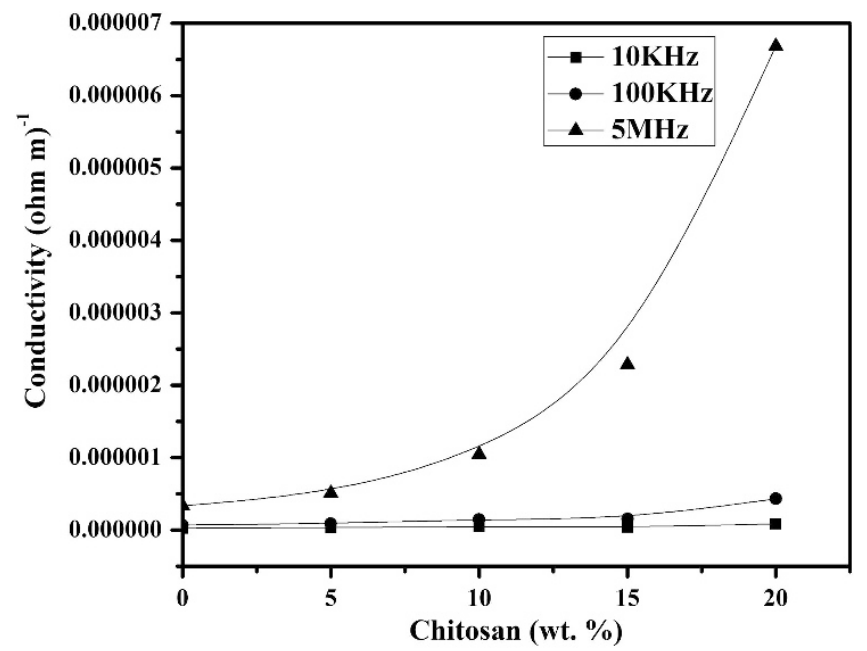

Figure 6 The effect of chitosan loading on the conductivity of plasticized composites films at various frequencies.
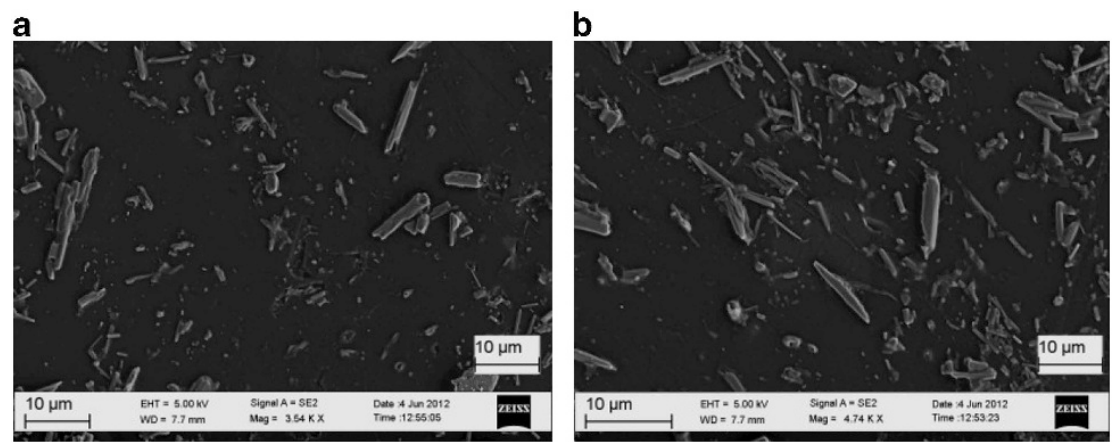

Figure 5 Scanning electron micrograph images of low-density polyethylene (LDPE)/15 wt\% chitosan composites with (a) unplasticized and (b) plasticized films. 


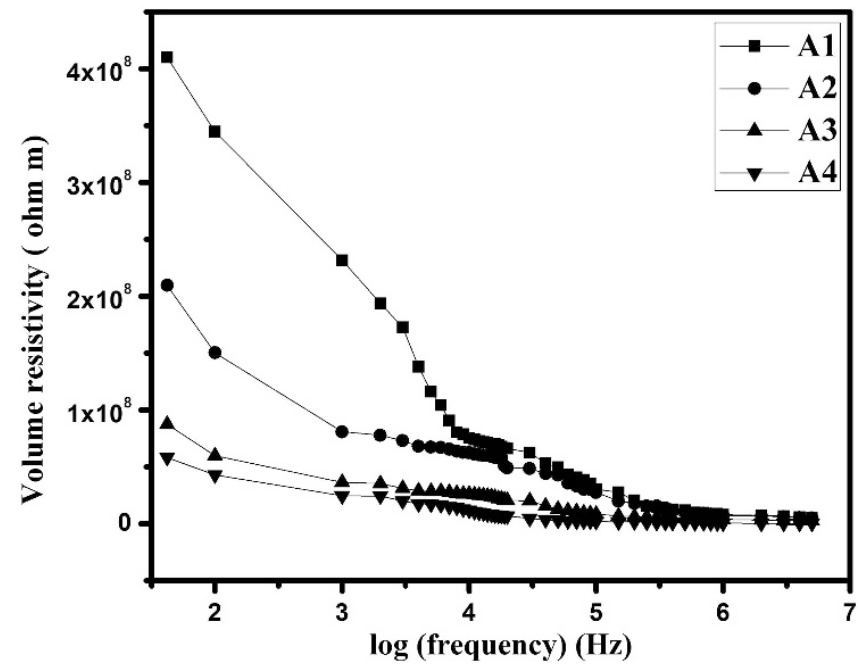

Figure 7 The effect of chitosan loading on volume resistivity as a function of frequency. A1, A2, A3 and A4 are the unplasticized low-density polyethylene (LDPE) composite films with $0,5,10$ and 15 wt\% chitosan loaded, respectively.

increased. This was due to the increment in the number of polar groups incorporated by chitosan loading. When loaded with chitosan, the fillers were more tightly packed and pressed against each other, which reduced the internal contact resistance and increased crystallinity. Thus, the net resistance (internal contact resistance and resistance of the fillers) decreased with increasing chitosan loading. When the contact resistance became very low, the aggregate resistance contributed to the net resistance at lower and higher frequencies. ${ }^{29,30}$ Thus, the composite systems crossed the percolation threshold and increased the conductivity because of a more conductive network with increasing chitosan loading.

The study of the volume resistivity $(\rho)$ of an insulating material is important because the most desirable property of an insulator is its ability to resist the leakage of electric current. $\rho$ Is the reciprocal of electrical conductivity. Figure 7 shows the plot of the volume resistivity of unplasticized films as a function of the logarithm of the frequency at different chitosan loadings. The volume resistivity decreased with an increasing frequency and filler concentration. As chitosan is hydrophilic in nature, they absorb moisture and contribute to the increased polarization. This resulted in a decreased volume resistivity with increased filler loading. It is well known that in any polymeric material, most of the charge transfer is through the crystalline regions, and the amorphous (non-crystalline) regions allow static charge transfer because of the presence of moisture. ${ }^{31,32}$ As suggested earlier, the polarity of the system increased with the incorporation of chitosan; it also increased because of the increased crystallinity. This increases the orientation polarization and the interfacial polarization, which results in an increase in the dielectric constant and a decrease in resistivity.

Figure 8 shows the dielectric constant of the sample with $15 \mathrm{wt} \%$ chitosan (B4) and its de-acetylated low-molecular-weight (nB4) composite film. From Figure 8, it can be observed that the system with LWCS showed a significant increase in the dielectric constant compared with its high-molecular-weight counterpart. In the case of LWCS particles, the total number of polar hydroxyl groups and $\mathrm{NH}_{3}+$ ions in the polymer chain increased, which led to increased dipoles in the LDPE/LWCS composite films. The size of the filler

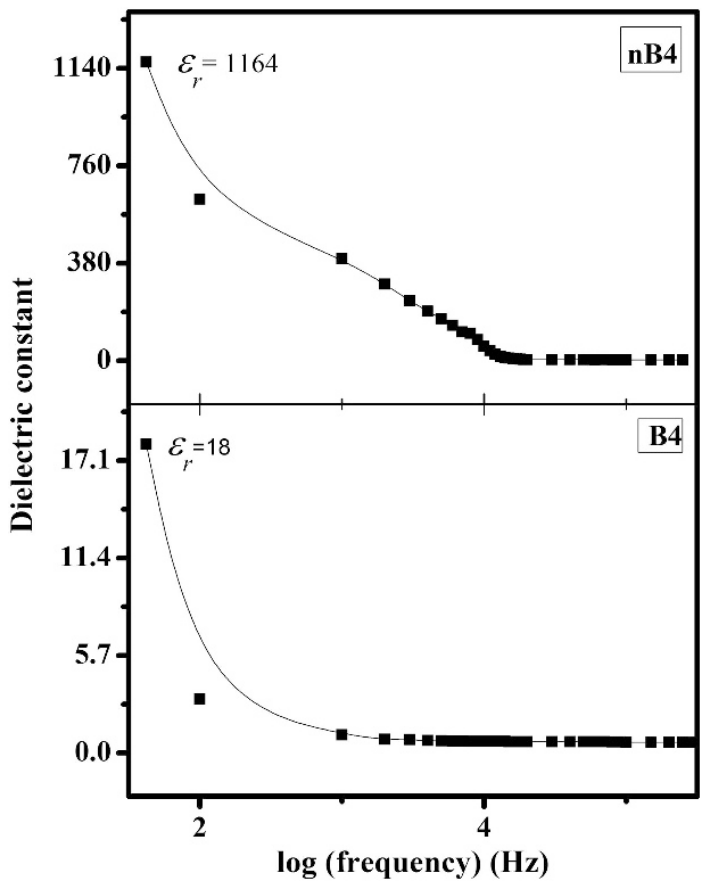

Figure $\mathbf{8}$ The effect of the molecular weight of chitosan on the dielectric constant of the composite films as a function of the logarithm of the frequency. B4 and nB4 are the film plasticized with $15 \mathrm{wt} \%$ chitosan and its de-acetylated low-molecular-weight chitosan (LWCS) composites, respectively.

particles also had a crucial role in the dielectric properties of the composite films. ${ }^{33,34}$ The size of the LWCS particles were smaller than the high-molecular-weight counterparts, which could be more clearly observed from the FESEM images (Figure 9). In the figure, the chitosan particles were spherical and were dispersed uniformly in the LDPE matrix. The dielectric constant increased linearly as the particle size of the filler decreased. This was because smaller particles with a higher surface area can provide better particle-to-particle contact. Owing to the close packing of fillers, the polarization arising from the dipole-dipole interaction was enhanced. The addition of LWCS particles enhanced the cationic charge density in the biocomposites, as revealed by the study on dielectric properties. This positive charge density enabled these composite films to interact with negatively charged microbial cell membranes, polymers, macromolecules and even with certain polyanions upon contact in an aqueous environment. This indicates that the significant charge interaction of the LDPE/LWCS films can be exploited for the antibacterial food packaging applications.

\section{Antimicrobial activity}

The dielectric property studies of the plasticized and unplasticized composite films revealed the important role of amino groups of chitosan, which furnished $\mathrm{NH}_{3}{ }^{+}$to the LDPE matrix. These positive ions interacted with the negatively charged cell walls of the bacteria and hindered the growth of such bacteria. ${ }^{35}$

The optical photographs in Figures 10a and b show the typical antimicrobial test results of the plasticized composite films (B1, B2, B3 and B4) against Escherichia coli and Staphylococcus aureus, respectively. The listed inhibitory activities were estimated from the area measurement of clear inhibition zones surrounding the film discs in the agar plates. The figures show negligibly small inhibition zones 

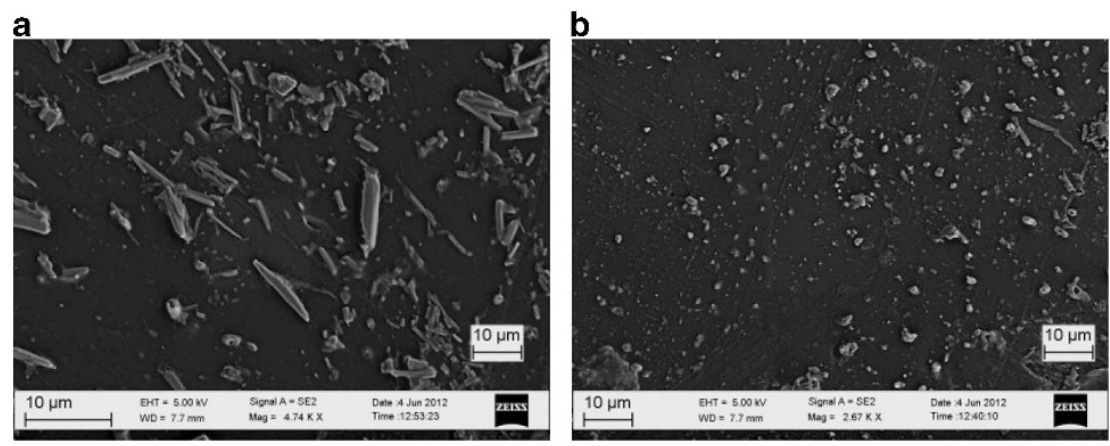

Figure 9 Scanning electron micrograph images of (a) low-density polyethylene (LDPE)/15 wt\% chitosan composite films and (b) LDPE/15 wt\% LWCS composite films.

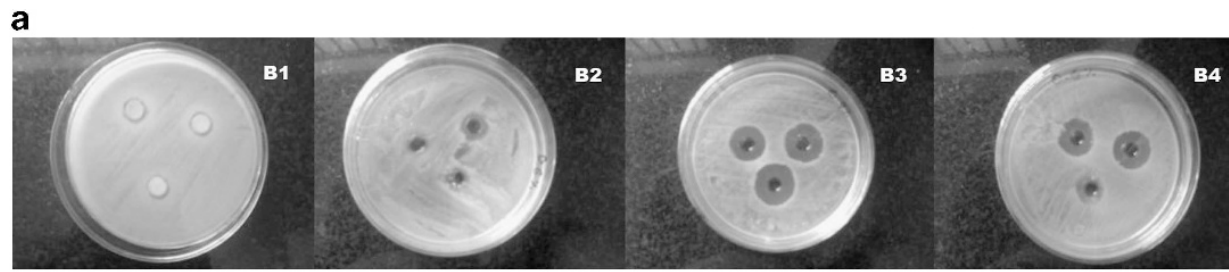

b

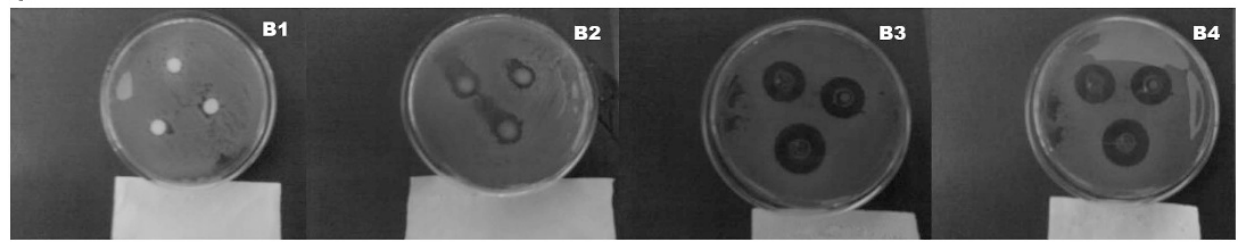

Figure 10 The results of the antimicrobial test of the plasticized composite films against (a) Escherichia coli and (b) Staphylococcus aureus.

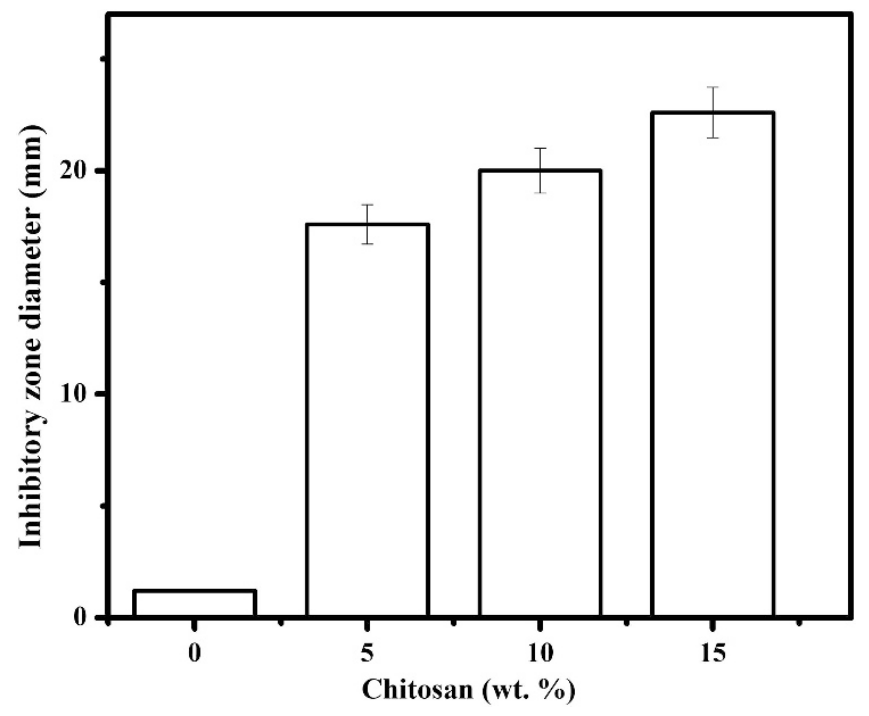

Figure 11 The effect of chitosan loading on the inhibitory zone development against Escherichia coli.

surrounding the pure LDPE film (B1). From the antimicrobial activity result of $\mathrm{B} 1$, it is clear that the additives plasticizer, maleic anhydride and dicumyl peroxide did not have a significant role in the antibacterial activity of the developed composite films. In the case of the chitosan-loaded composites, however, distinct inhibitory zones developed surrounding the films. This inhibitory action was due to the antibacterial property of the chitosan present in the film. The antibacterial properties of chitosan were attributed to the electrostatic interaction between the electronegative microbial surfaces and the positively charged chitosan. This charge interaction led to microbial cell wall damage and the leakage of the intracellular constituents. This, in turn, led to the binding of chitosan molecules with DNA and the inhibition of mRNA synthesis in the microbial organisms. ${ }^{36-38}$ The experimental inhibition area for the various plasticized composite films against Escherichia coli are shown in Figure 11. It can be observed from the figure that the diameter of the inhibition zone increases with chitosan loading. This is due to the increase in charge density $\left(\mathrm{NH}_{3}{ }^{+}\right)$with the increase in chitosan content, which was also observed in the dielectric property studies. There was significant microbial cell wall inhibition that took place with the chitosan loads in the composite systems. ${ }^{39,40}$ However, such charge density cannot be observed in the pure LDPE film and led to a smaller inhibitory zone. The data from the limited number of microorganisms tested showed that the inhibitory activity of LDPE-chitosan composite films was restricted to Gram-positive and Gram-negative species.

Figure 12 shows the antibacterial test results of B4 and nB4 films against Escherichia coli. The LDPE/LWCS composite films exhibited more distinctive inhibitory zones with larger diameters against microbial organisms than its high-molecular-weight counterpart. This is because of the high polycationic nature of the LWCS-based films, as revealed by the dielectric property study 

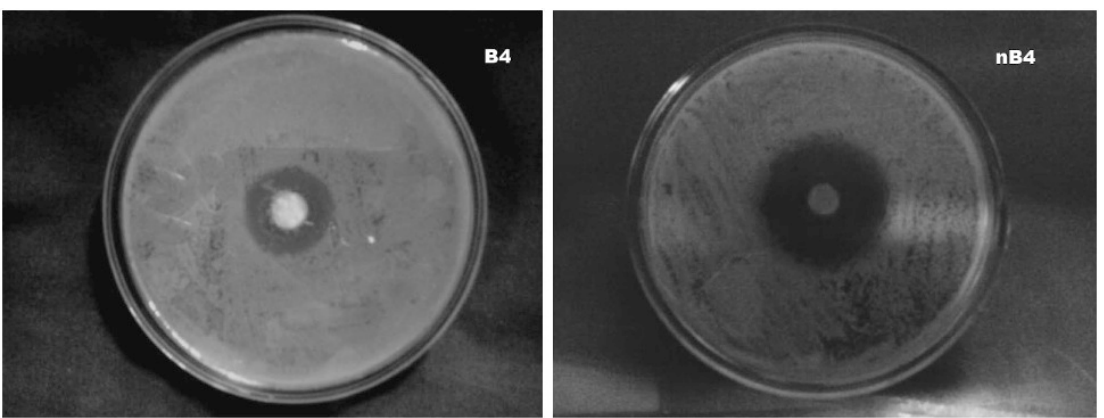

Figure 12 The results of antibacterial tests of the B4 and nB4 films against Escherichia coli.

(Figure 8). The antimicrobial activity of the tested samples is in accordance with their dielectric behavior. This correlation will be helpful for the assessment of the antibacterial activity of the biocomposite films with polar fillers without a biological test. In addition, the dielectric property measurement can be considered a crucial tool for ensuring the antimicrobial activity of the eco-friendly food packaging films.

\section{CONCLUSIONS}

The newly developed plasticized LDPE-chitosan film is a promising food packing material with excellent antimicrobial activity. The dielectric properties, such as the dielectric constant, volume resistivity and conductivity, of the LDPE-chitosan composite films were studied as a function of the filler content, applied AC frequency and the presence of plasticizer. The results were correlated with the antibacterial activity of the film. The maximum values of the dielectric constant in the lower frequency region have been attributed to interfacial polarization. An increase in the dielectric constant of composites with filler loading was due to the increased orientation and interfacial polarizations. The addition of palm oil as a plasticizer increased the segmental motion of the polymeric chains and filler dispersion in the matrix, which further increased the dielectric constant of the composites. The volume resistivity of the composites was found to decrease with chitosan loading. The conductivity of the composites was also found to increase with filler loading. The study on antimicrobial activities showed that plasticized LDPE-chitosan composite films developed an inhibitory zone against Escherichia coli and Staphylococcus aureus, and the zone diameter increased with the chitosan content in the films. This was due to the electrostatic interaction between the negatively charged microbial cell membrane and the positively charged chitosan in the composites. The effect of the molecular weight of chitosan on the dielectric and antimicrobial properties of the composites were also studied in this work. It was found that the dielectric constant and the antibacterial properties of the samples increased as the molecular weight of chitosan decreased. The antibacterial properties of the developed films were determined to be complementary with their dielectric properties. This study highlighted the possibility of using the dielectric properties of composites as a measure of their antibacterial properties.

\section{ACKNOWLEDGEMENTS}

We are grateful to the Kerala State Council for Science and Technology and Environment (616/2012/KSCSTE), Thiruvananthapuram, Kerala, India for awarding a project under this subject area. We thank Mr KS Gopi and Miss K Sreebha, MRCMPU Ltd, MILMA, Kerala, India, for the antimicrobial studies.
1 Hotchkiss, J. H. Food packaging interactions influencing quality and safety. Food Addit. Contam. 14, 601-607 (1997).

2 Han, J. H. Antimicrobial food packaging. Food Technol. 54, 56-65 (2000).

3 Rios, J. L. \& Recio, M. C. Medicinal plants and antimicrobial activity. J. Ethnopharmacol. 100, 80-84 (2005).

4 Quintavalla, S. \& Vicini, L. Antimicrobial food packaging in meat industry. Meat Sci. 62, 373-380 (2002).

5 Cooksey, K. Effectiveness of antimicrobial food packaging materials. Food Addit. Contam. 10, 980-987 (2005).

6 Brody, A. Active Packaging: Beyond Barriers (Packaging Strategies, West Chester PABRG Townsend, Mt. Olive, NJ, 2002).

7 Takagi, H. \& Asano, A. Effects of processing conditions on flexural properties of cellulose nanofiber reinforced green composites. Compos. Part A 39, 685-689 (2008).

8 Akil, H. M., Omar, M. F., Mazuki, A. A. M., Safiee, S., Ishak, Z. A. M. \& Bakar, A. A. Kenaf fiber reinforced composites: a review. Mater. Des. 32, 4107-4121 (2011).

$9 \mathrm{Hu}$, S. G., Jou, C. H. \& Yang, M. C. Protein adsorption, fibroblast activity and antibacterial properties of poly (3-hydroxybutyric acid-co-3-hydroxyvaleric acid) grafted with chitosan and chitooligosaccharide after immobilized with hyaluronic acid. Biomaterials 24, 2685-2693 (2003).

10 Li, B., Huang, L., Wang, X., Ma, J. \& Xie, F. Biodegradation and compressive strength of phosphorylated chitosan/hydroxyapatite bio-composites. Mater. Des. 32, 4543-4547 (2011)

11 Young, D. H. \& Kauss, H. Release of calcium from suspension-cultured Glycine max cells by chitosan, other polycations, and polyamines in relation to effects on membrane Permeability. Plant Physiol. 73, 698-702 (1983).

12 Naficy, S. \& Garmabi, H. Study of the effective parameters on mechanical and electrical properties of carbon black filled PP/PAG microfibrillar composites. Compos. Sci. Technol. 67, 3233-3241 (2007).

13 Radhakrishnan, S. \& Saini, D. R. Structure and dielectric properties of poly (viny chloride) thermoplastic elastomer blends. J. Appl. Polym. Sci. 1577-1586 (1994).

14 Mansour, A. A., El-Sabagh, S. \& Yehia, A. A. Dielectric investigation of SBR-NBR and CR-NBR blends. J. Elastom. Plast. 26, 367-378 (1994).

15 Schifani, R., Spadaro, G., Cassata, F. \& Valenza, A. Dielectric behaviour of LLDPE/PC blends. Eur. Polym. J. 31, 841-846 (1995).

$16 \mathrm{Hampl}$, J. \& Bouda, V. I-V characteristics of the carbon black-filled polyethylene interface with tin electrodes. Synthetic Met. 67, 129-132 (1994).

17 George, S., Varughese, K. T. \& Thomas, S. Dielectric properties of isotactic polypropylene/nitrile rubber blends: effects of blend ratio, filler addition, and dynamic vulcanization. J. Appl. Polym. Sci. 73, 255-270 (1999).

18 Du, W. L., Niu, S. S., Xu, Y. L., Xu, Z. R. \& Fan, C. L. Antibacterial activity of chitosan tripolyphosphate nanoparticles loaded with various metal ions. Carbohdr. Polym. 75, 385-389 (2009).

19 Tharanathan, R. N. Chitin-the undisputed biomolecule of great potential. Crit. Rev. Food Sci 43, 61-87 (2003).

20 Ozdemir, M. \& Floros, J. D. Active food packaging technologies. Crit. Rev. Food Sci. 44, 185-193 (2004)

21 Ferreira, L. M., Falcao, A. N. \& Gil, M. H. Modification of LDPE molecular structure by gamma irradiation for bio applications. Nucl. Instrum. Meth. B 236, 513-520 (2005).

22 Sunilkumar, M., Francis, T., Thachil, E. T. \& Sujith, A. Low density polyethylenechitosan composites: a study based on biodegradation. Chem. Eng. J. 204-206, 114-124 (2012)

23 Brydson, J. Plastics Materials. 7th edn (Elsevier publishers, Elsevier India Private Limited, New Delhi, India, 2005).

24 Pranoto, Y., Salokhe, V. \& Rakshit, K. S. Physical and antibacterial properties of alginate-based edible film incorporated with garlic oil. Food Res. Int. 38, 267-272 (2005)

25 Breukink, E. \& Kruijff, B. D. The lantibiotic nisin, a special case or not. Biochim Biophys. Acta 1462, 223-234 (1999).

26 Jydegaard, A. M., Gravesen, A. \& Knochel, S. Growth condition related response of Listeria monocytogenes 412 to bacteriocin inactivation. Lett. Appl. Microbiol. 31, 68-72 (2000). 
27 Bicerano, J. Prediction of Polymer Properties, Chapter 9 (Marcel Dekker Inc., New York, 1993).

28 Smyth, C. P. Dielectric Behaviour and Structure p. 53 (McGraw Hill, Oxford, 1956).

29 Rajesh, C., Manoj, K. C., Unnikrishnan, G. \& Purushothaman, E. Dielectric properties of short nylon -6 fiber -reinforced NBR composites. Adv. Polym. Tech. 32, 90-102 (2011).

30 Wang, J. \& Zhao, K. Dielectric analysis of chitosan-iron composite microspheres suspensions: access to internal and interface electrokinetic information of microspheres. Colloid. Surface A 396, 270-277 (2012).

31 Legge, N. R., Holden, G. \& Schoeder, H. E. Thermoplastic Elastomers-A Comprehensive Review (Hanser Publishers, New York, 1987).

32 Linford, R. G. Electrical and Electrochemical Properties of Ion Conducting Polymers, in Applications of Electroactive Polymers p. 1-28 (Chapman and Hall, B. Scrosati, London, 1993).

33 Lewis, T. J. Interfaces are the dominant feature of dielectrics at the nanometric level. IEEE Dielect. El. In 11, 739-753 (2004).
34 Lewis, T. J. Interfaces: nanometric dielectrics. J.Phys. D: Appl. Phys. 38, 202-212 (2005).

35 Zheng, L. Y. \& Zhu, J. F. Study on antimicrobial activity of chitosan with different molecular weights. Carbohydr. Polym. 54, 527-530 (2003).

36 Sagoo, S., Board, R. \& Roller, S. Chitosan inhibits growth of spoilage microorganisms in chilled pork products. Fd. Microbiol. 19, 175-182 (2002).

37 Nadarajah, K., Prinyawiwatkul, W., No, H. K., Sathivel, S. \& Xu, Z. Sorption behaviour of crawfish chitosan films as affected by chitosan extraction processes and solvent types. J. Fd. Sci. 71, 33-39 (2006).

38 Muzzarelli, R. A. A. \& Rocchetti, R. Determination of the degree of acetylation of chitosan by first derivative ultraviolet spectrophotometry. Carbohydr. Polym. 5, 461-472 (1985).

39 Liu, N., Chen, X. G., Park, H. J., Liu, C. G., Liu, C. S. \& Meng, X. H. Effect of MW and concentration of chitosan on antibacterial activity of Escherichia coli. Carbohydr. Polym. 64, 60-65 (2006).

40 Maghami, G. G. \& Roberts, G. A. F. Evaluation of the viscometric constants for chitosan. Makromol. Chem. 189, 195-200 (1988). 